\title{
The Effect of Saffron Aqueous Extract and Crocin on PTSD Rat Models: The Focus on Learning and Spatial Memory
}

\author{
Sara Asalgoo1 $^{(i D}$, Gila Pirzad Jahromi*2 ${ }^{\text {(D) }}$, Boshra Hatef ${ }^{2}$ (D), Hedayat Sahraei ${ }^{\text {(D) }}$ \\ 1. Behavioral Sciences Research Center, Baqiyatallah University of Medical Sciences, Tehran, Iran \\ 2. Neuroscience Research Centre, Baqiyatallah University of Medical Sciences, Tehran, Iran
}

\begin{tabular}{|c|}
\hline Article Info \\
\hline dol $10.30699 /$ jambs.26.119.34 \\
\hline $\begin{array}{l}\text { Received: 2018/06/27; } \\
\text { Accepted: 2018/08/24; } \\
\text { Published Online: 01 Nov 2018; }\end{array}$ \\
\hline $\begin{array}{l}\text { Use your device to scan and read the } \\
\text { article online }\end{array}$ \\
\hline 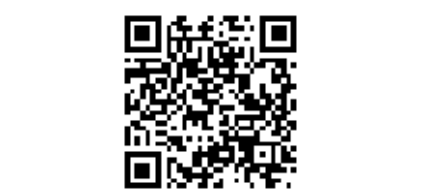 \\
\hline
\end{tabular}

Corresponding Information: Gila Pirzad Jahromi, Neuroscience Research Centre, Baqiyatallah University of Medical Sciences, Tehran, Iran Email: gpirzad1340@gmail.com

\begin{abstract}
Background \& Objective: Post traumatic stress disorder (PTSD) is an anxiety reaction, which occurs as a result of encountering a seriously traumatic event during one's lifetime. The aim of this study was to evaluate the effect of saffron aqueous extract and crocin on spatial memory and learning with the Barnes maze in a PTSD model on male Wistar rats (Weighting 200-250 gr)
\end{abstract}

Materials \& Methods: Wistar rats $(\mathrm{n}=48)$ were randomly divided into two groups: PTSD and non-PTSD. The PTSD group first received intra-cerebero-ventricular (ICV) administration of $10 \mu \mathrm{g} / \mathrm{rat}$ aqueous saffron extract, crocin or saline and then an electric foot shock. After 21 days, both groups were returned to the electric shock box in order to remember stressors without receiving any shocks. Corticosterone levels were then measured in the samples. Concurrently, a digital camera was recording the animals' behaviors. Upon this, spatial learning and memory was assessed for five consecutive days.

Results: The saffron extract and crocin caused a significant increase $(P<0.001)$ in corticosterone levels and a significant reduction $(P<0.05)$ in freezing behavior, as well as a significant difference $(P<0.001)$ in spatial learning of the two groups.

Conclusion: Our results indicated the potential role of saffron aqueous extract and its active derivative (crocin) in improving behavioral symptoms and spatial learning in PTSD models.

Keywords: Traumatic stress disorder, Crocin, Corticosterone, Spatial learning, Spatial memory

\section{Introduction}

Psychological trauma caused by life-threatening events can cause serious physical injuries in an individual (1). Although, these experiences might sometimes be associated with severe fear, panic, and disappointment, which can gradually lead to anxiety disorders such as post-traumatic stress disorder (PTSD) (2). The hypothalamic pituitary adrenal (HPA) axis is the first line of defense against stress-induced conditions in the body. Under stress, Corticotrophin releasing hormones (CRH) is released from the hypothalamic paraventricular nucleus (PVN), leading to the release of ACTH from the anterior pituitary, which ultimately leads to the release of glucocorticoids from the adrenal gland (3). It should be noted that PTSD is determined by the reduction of plasma cortisols, the increase of corticotrophin releasing hormones $(\mathrm{CRH})$ in the cerebrospinal fluid and plasma, and the increase of negative feedback in the pituitaryadrenal-axis suppression (4). In fact, there is a correlation between various parts of the brain and the HPA axis in stressful situations. There is a two-way relationship between the amygdule and the paraventricular nucleus (PVN) of the hypothalamus neurons. The neurons of the hypothalamus are responsible for the secretion of $\mathrm{CRH}$ in the brain and in neurotransmitters such as glutamate, dopamine, noradrenaline, and opioids involved in the brain's stressor response (5).

One of the most obvious symptoms of PTSD is a learning disorder which surfaces during and after the occurrence of a traumatic event. Studies show that during a traumatic experience, a person refuses to recall the signs that reminds him of the traumatic experience. For this reason, the person is constantly in a state of anxiety (6). Chronic anxiety is known as a pathological disorder, which is also associated with learning disabilities in people with PTSD, and is often accompanied with an absence of security (7). Another study has shown that PTSD patients are often confronted with problems such as concentration, attention, and memory impairment (8). In another study, the reduction in concentration had been reported by the decrease in the volume of the hippocampus in PTSD patients and war victims (9). The hippocampus region, which includes areas such as the dentate gyrus and $\mathrm{CA}$, is an important anatomical structure in the field of human memory. Therefore, damage to this area results in learning and memory 
impairment (10). In different studies, the single-cell recording and neuronal imaging techniques have reported changes in the category of neurons in the hippocampus, along with the changes in learning and memory retention (11). Negative stimuli can cause changes in brain data encoding and retrieval. Neurohormones, such as norepinephrine and cortisol, have a significant effect on the response to fear and stress through the HPA axis and the amygdual and play an important role in responding to fear and stress in the form of body freezing. Additionally, Norepinephrine improves emotional memory (12).

Saffron (Crocus sativus L.) is a perennial plant (from the lily family), known as red gold, as its cultivation is very costly. Flowers of Crocus sativus L. are bright purple and its stigma is fairly red. The flower stigma not only contains a nutritional value but is considered as a spice. It should be noted that saffron has been considered as great importance in traditional medicine for many years (13). In the current day, the usage of this plant is used extensively to treat diseases such as insomnia, depression, cognitive impairment, seizure, cardiovascular disorders, cancer, trauma, anxiety, and fatigue (14). Crocin is a main pigment of saffron which has anti-inflammatory, antiurethral, anti-epileptic analgesic effects. It also contains strong antioxidant properties which aid in the increase of nerve regeneration and microbial polymerization. Additionally, crocin has the ability to improve chronic cerebral hypoperfusion and learning and memory impairments, which occur due to the effects of chronic stress (15). According to a study, in the in vitro environment, saffron has a significant effect on NMDA receptors and prevents NMDA receptors of a rat's hippocampal neurons to be affected by ethanol inhibitors (16). Furthermore, saffron and its effective ingredients, such as safranal and crocin, correct the inhibitory effects of morphine on spatial learning and the harmful effects of scopolamine on memory $(17,18)$. These results indicate that saffron and its effective ingredients have an effect on the storage and retrieval of memory data and may be useful in the improvement of neurodegenerative diseases such as Alzheimer's disease $(19,20)$.

Hence, the purpose of this study was to investigate the effects of saffron aqueous extract and crocin on spatial learning and memory in PTSD models of adult male rat using Barnes maze. To this end, an electric foot shock box was used to create a PTSD model and to perform ICV injections of saffron extract. Upon creating a PTSD model, Corticosterone concentrations were measured at baseline, stress, and final levels. Behavior symptoms, such as freezing, were also investigated.

\section{Materials and Methods}

\section{Saffron Aqueous Extract Preparation}

In this study, we used the stigmas of Crocus sativus made from Talakaran-e-Mazra'eh Company (Torbat Heydarieh, Khorasan, Iran). At first, $100 \mathrm{~g}$ of dried stigma was dissolved in $1000 \mathrm{~mL}$ of distilled water, maintain a 30 to $35^{\circ} \mathrm{C}$ temperature for evaporation. In the extraction process, $24 \mathrm{~g}$ of dried powder was finally obtained from $100 \mathrm{~g}$ of stigma. The extract of $10 \mu \mathrm{g} / \mathrm{rat}$ was immediately injected into the left lateral ventricle of the rat's brain 5 minutes previous to receiving the shock (21).

\section{Description of Chemical Compounds in Saffron Extract}

The compound of saffron stigma was analyzed by Hosseinzadeh and Jahanian in 2010 with a number of reforms (ISO 3632-2). The chemical compounds above were read and analyzed on the basis of direct absorption. According to the analysis, picrocine was a bitter caustic agent in saffron at $270 \mathrm{~nm}$ and in dry conditions at 96.6 $\mathrm{nm}$, safranal was the source of saffron flavor at $330 \mathrm{~nm}$ and in dry condition at 41.5, and finally, crocin was the main combination and color source of saffron at $440 \mathrm{~nm}$ and in dry conditions at $259 \mathrm{~nm} \mathrm{(22).}$

\section{Animals}

The current study was permitted by the ethics committee of Baqiyatallah University of Medical Sciences, Tehran, Iran (ID: IR.BMSU.REC.1395.380). In this study, adult Wistar rats, weighing 230-250 g, were used. The animals were taken from the experimental breeding center of Pasteur Institute one week before the experiments and were transferred to the animal lab for environment adaptation in special cages of 3-4 groups. The rats were provided with sufficient water and food at all times, except during the experiment. They were kept at $22 \pm 2^{\circ} \mathrm{C}$ and alternate 12/12-hour dark/light. After 28 days, the tests were carried out during the day under standard conditions. Then, animals were randomly divided into 2 groups, including PTSD and non-PTSD models. The former group was divided into 4 subgroups ( $\mathrm{n}=8$ per group): 1 stress receiving group without medication $\left(\mathrm{Ctrl}^{+}\right)$, 2stress receiving group with normal saline (PTSD-saline), and 3-stress receiving group with medication of saffron extract (Treat-saffron) or crocin (Treat-crocin) (sigma17304, CAS-No: 42553-65-1)). The non-PTSD group was also divided into 2 subgroups ( $n=8$ per group): Negative control group (without surgery, $\mathrm{Ctrl}^{-}$) and placebo group (Only the scalp was opened) (Figure 1).

\section{Surgery Method}

Hydrochloride (70 $\mathrm{mg} \mathrm{kg}^{-1}$, i.p.) and xylazine $(10 \mathrm{mg}$ $\mathrm{kg}^{-1}$, i.p.) were used to anesthetize the animals. The guide cannula (23-gauge), made of stainless steel, was placed in the left ventricle of the brain by stereotaxic apparatus (stoelting instruments, USA), and its location was determined using the coordinates presented in the Paxinos \& Watson atlas (1987). This location is - 0.9 $\mathrm{mm}$ posterior of the bergman, $1.5 \mathrm{~mm}$ of the sagittal, $3.5 \mathrm{~mm}$ from dura matter of upper cranium and $-3.3 \mathrm{~mm}$ from incisor bar location. The cannula was then tightened with dental cement on the skull. 


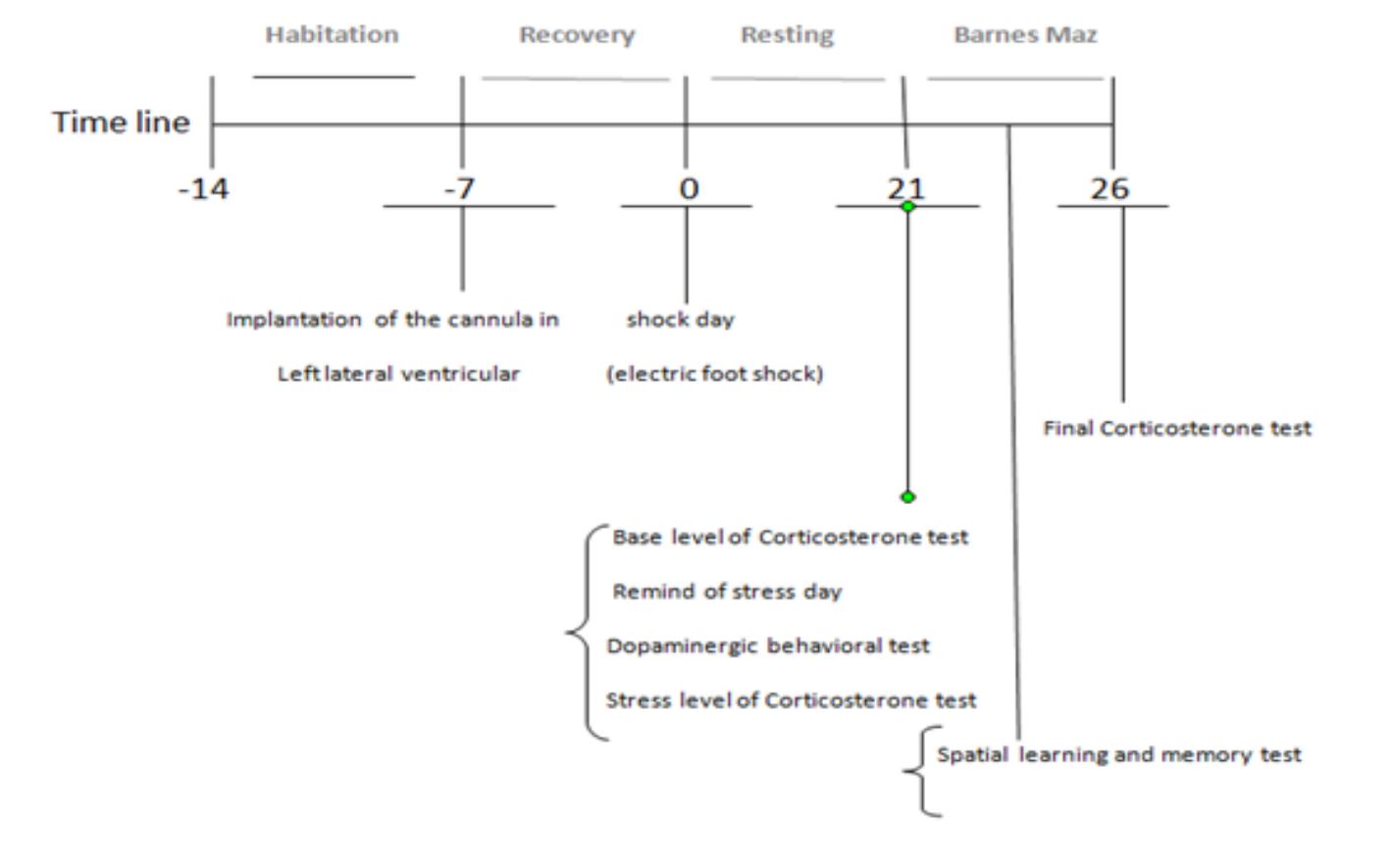

Figure 1. Protocol Time line

\section{The Creation of a PTSD Model in an Animal by Induction of Electric Foot Shock}

Here, we used an electric shock box to create a PTSD model in an animal. The electric shock box is made from transparent Plexiglas by Borj-e-Sanat Co., consisting of 9 separate compartments $(16 \times 16 \mathrm{~cm})$ with a stainless steel rod (4 $\mathrm{mm}$ in diameter), at interval of $2 \mathrm{~cm}$. There are some holes of $2.5 \mathrm{~cm}$ diameter in the walls of the box for communication between olfaction and audio. The floor of the chamber was composed of bars that connected to the electric generator, through which the intensity and duration of the induced shock could be altered. The electric shock, applied in this study, was 2 $\mathrm{mA}$ shocks for the duration of 1 second and repeated upon every 30 seconds. Therefore, each rat was delivered 10 shocks in a 5 minutes time span in total $(15$, 23, 24).

\section{Evaluating the Level of Corticosterone Through the ELISA Kit}

Twenty one days after receiving the shocks, blood samples were collected from the orbital sinus in a volume of $2 \mathrm{~mL}$ between 12:00-14:00, both before being placed in the shock box to remind of stress (base level) and after they were placed in the shock box to remind of stress (stress level). On day 26, the final level of the evaluation of return to base levels of corticosterone was measured. Then, the samples were centrifuged at 3000 rpm for 8 minutes at $4^{\circ} \mathrm{C}$. Supernatant was also collected and measured using ELISA DRG Corticosterone kit (Cat. Nr EIA 4164) in $450 \mathrm{~nm}$.

\section{Evaluating Freezing Behavior}

Animals of both groups (PTSD and non-PTSD) were returned to the electrical box 21 days after receiving shocks for the duration of 5 minutes to be reminded of stressors. During this time, the animal did not receive any shocks. Dopamine-related behaviors such as freezing (copying behavior in seconds) were recorded by a digital camera $(\mathbf{1 5}, 24)$.

\section{Spatial Learning and Memory Assessment with Barnes Maze}

On day 22, spatial memory and learning was evaluated in this study, through a circular table made of transparent Plexiglas of $95 \mathrm{~mm}$ in diameter and 12 holes of $10 \mathrm{~mm}$ in diameter. A chamber made of dark Plexiglas with 20 x $20 \mathrm{~cm}$ dimension was placed beneath one of the holes (target hole). In order to guide the animal to find the target's hole, a light source of 500 watts was installed at $100 \mathrm{~cm}$ from the maze table. One day before the test, each animal was placed on the maze table for 60 minutes in order to be trained. On the first day, the animal was held in the middle of the table and a dark Plexiglas, $15 \times 15 \mathrm{~mm}$ in diameter, was placed upon it. The light was turned on after 10 seconds of the placement and until a given 90 second timespan in order for the animal to find the target hole. If the animal had failed to find the target hole, it would have been guided by hand. After finding the hole, the target hole was covered by a dark screen and the animal was held there for 60 seconds. This test was carried out in 4 daily trials during 4 days. The animal's memory was then evaluated on day 5 . Between each trial, the maze table was cleaned with $70 \%$ ethanol to eliminate the effects of odor on the surface of the maze. In this study, indicators such as latency time (time of animal movement from the center of the table until the target hole), number of errors in arrival at the target hole (number of times a head or nostril enters a non-target hole) and distance (traveled distance on the maze table 
to reach the target hole) were also evaluated. All of the Barnes maze information was recorded by a digital camera. Additionally, the strategy used by the rats in each trial was recorded for 4 consecutive days. These strategies included the serial strategy (the search method is rotated clockwise or counterclockwise to find the target hole), random (non-continuous and unorganized search from the middle of the Barnes table to find the target hole), and direct search (direct movement to the target hole or inside the two adjacent holes before visiting the target hole) (25).

\section{Data Analysis}

One-way analysis of variance and Tukey's post hoc tests were used to compare intergroup mean \pm SEM. In addition, one-way analysis of variance and Tukey's post hoc test were run to evaluate freezing behavior, in order to compare the groups so that $\mathrm{P}$-value $<0.05$ was considered significant. The mixed-model ANOVA test was also used to compare corticosterone concentrations at three levels (basal, stress and final) due to the normal distribution among the groups. 2-way mixed model ANOVA was also used for analyzing quantitative variables such as the number of errors, latency time, and traveled distance, due to their normal distribution. Furthermore, the Kruskal-Wallis test was used to compare the distribution of search strategies between groups, and the Mann-Whiney test was used to compare pairwise groups.

\section{Results}

\section{Determination of the Surgical Location}

To evaluate the location of the cannula, $10 \mu \mathrm{g} / \mathrm{rat}$ of trypan blue was injected into the left lateral ventricular of the rat's brain, using a Hamilton syringe, and then the tissue samples were cut off and removed from the brain. The pathway injection in the left lateral ventricle of the brain was identified as blue.

The Effect of Saffron Aqueous Extract and Crocin on the Corticosterone Concentration

The results illustrated that there was no significant difference within each group of corticosterone concentration at basal, stress and final levels, yet, our results showed a significant decrease in the $\mathrm{Ctrl}^{+}$and PTSD- saline compared to the $\mathrm{Ctrl}^{-}$group $(P<0.05)$ at all three levels. Also, the results of the treatment group with crocin and saffron aqueous extract groups showed a significant increase in corticosterone concentration at all three levels compared to the $\mathrm{Ctrl}^{+}$and PTSD- saline groups with $(P<0.003)$ and $(P<0.01)$ respectively (Figure 2A). However, there was no significant difference between the treatment group with crocin and saffron and the $\mathrm{Ctrl}^{-}$group.

The Effect of Saffron Aqueous Extract and Crocin on Freezing Behavior

According to the results, while there was a significant increase in the freezing behavior of the PTSD groups $\left(\mathrm{Ctrl}^{+}(153.7149 .12\right.$ per seconds) $)$ and PTSD- saline groups (149.85 \pm 41.02 seconds) compared to the $\mathrm{Ctrl}^{-}$ group (10 \pm 3.46 seconds), $(P<0.05)$, a significant reduction has been observed in the freezing behavior of the treatment groups (Treat-saffron (18.42 \pm 3.8 seconds) ) and Treat-crocin ( $24 \pm 4.45$ seconds) compared to the $\mathrm{Ctrl}^{+}$and PTSD- saline groups $(P<0.05)$ (Figure 4). In this study, there was no significant difference between the freezing behavior of the treatment groups (saffron aqueous extract) and crocin compared to the negative control group (Figure 2B).
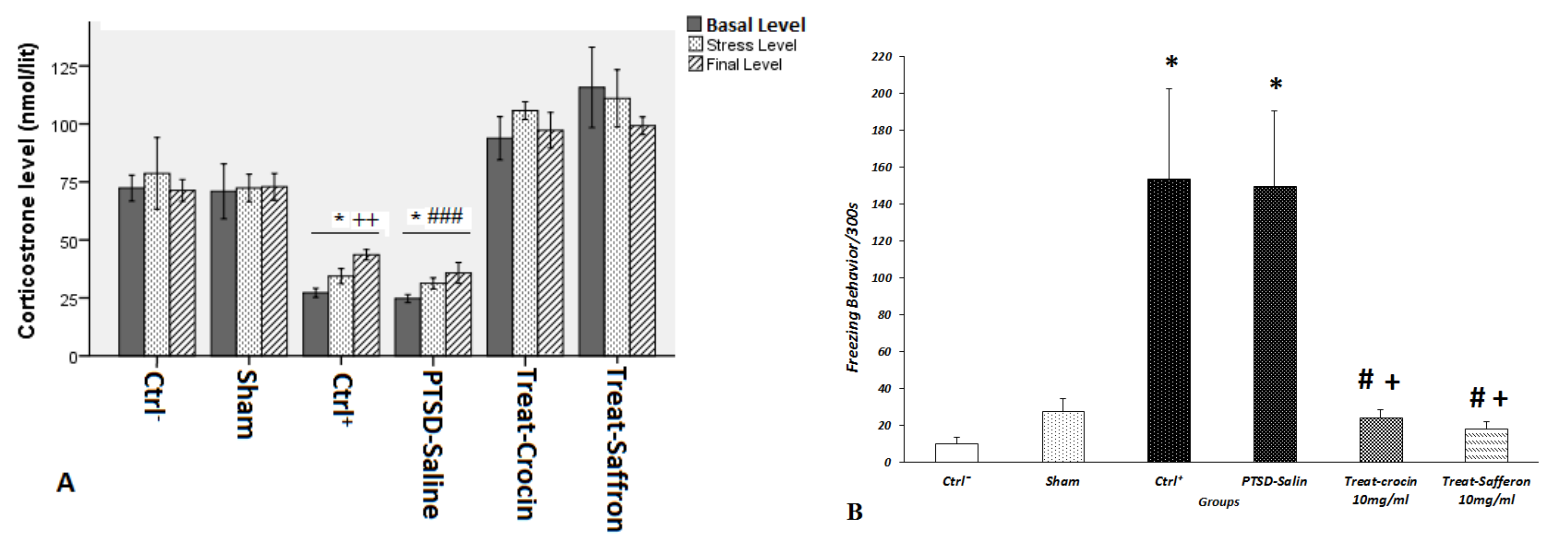

Figure 2. A: The evaluation of corticosterone concentration at three basal (before re-exposure), stress (re-exposure) and return to basal levels (after re-exposure) in between groups. $* P<0.5$ was a significant decrease in the positive control and PTSD-saline groups, compared to the negative control group. $++P<0.01$ was significantly different from the saffron group and \#\#\#P<0.001 was significantly different from Crocin group. B: Changes in the freezing time; the exploratory behavior was measured on day 21 post-shock in 6 groups at the re-exposure time. $* P<0.05$ was significantly different from the negative control group; ${ }^{+} P<0.05, \# P<0.05$ was significantly different from the positive control and PTSD-saline groups 


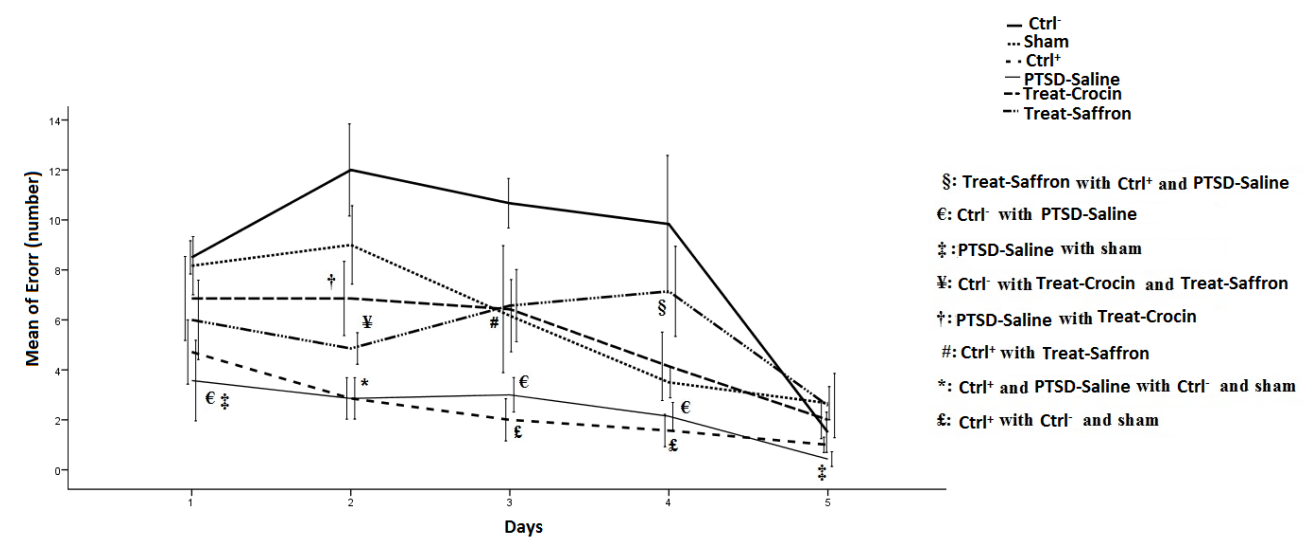

Figure 4. The effects of ICV administration of saffron aqueous extract on the number of errors to reach the target hole in rats, 21 days after electric foot shock stress delivery. There was a significant difference between the groups $(P<0.000007)$.

The Effect of Saffron Aqueous Extract and Crocin on Search Strategies

In relation to the search strategy analysis, the kaluskiewer test was used to evaluate the selected strategies in the groups during 4 days. Our results manifested the similarity of the same selective strategy between two groups of positive and PTSD-normal saline, as well as the behavioral similarity between the treatment groups with crocin and saffron. Therefore, the distribution analysis of three types of strategies were performed in regards to negative, sham, stress and treatment groups. During the 4 days of experiment, the significant levels of two-way kaluskiewer were 0.006, $0.016,0.000$ and 0.013 respectively. According to the results, all three strategies (direct, random and serial) were observed in the negative control group. However, none of these strategies had dominance over the negative control group on these 4 days. In the sham group, the dominant serial strategy was with a random and direct strategy. In addition, changes were also observed in the direct and random strategy groups on the 4 days. In the stress group, the random strategy was mostly used compared to the other groups, although the direct strategy increased over a period of 4 days. In the treatment group, the searched strategy was similar to the control group, with less random and more serial than the stress group (Figure3).

\section{Effect of Saffron Aqueous Extract and Crocin on the Number of Errors}

Based on the results of the compared variables between the groups of the maze test, the number of errors during the 5 days were reduced. Notably, this decrease is in conflict with the grouping $(P<0.05)$, so that, in comparison with the group in the negative, sham, and crocin groups, there was a significant decrease in the number of errors in finding the target hole, especially on the fifth day, while it was not observed in the positives and PTSD-saline and saffron groups. Yet, there was a significant difference between the groups $(P<0.000007)$. The differences between the groups during the days were evaluated based on the Mann-Whitney test. The results showed that the positive and PTSD-saline groups had significantly less number of errors than the negative and sham groups. Two treatment groups, especially the saffron group, tended to show similar behaviors as the control groups from day 3 (Figure 4).

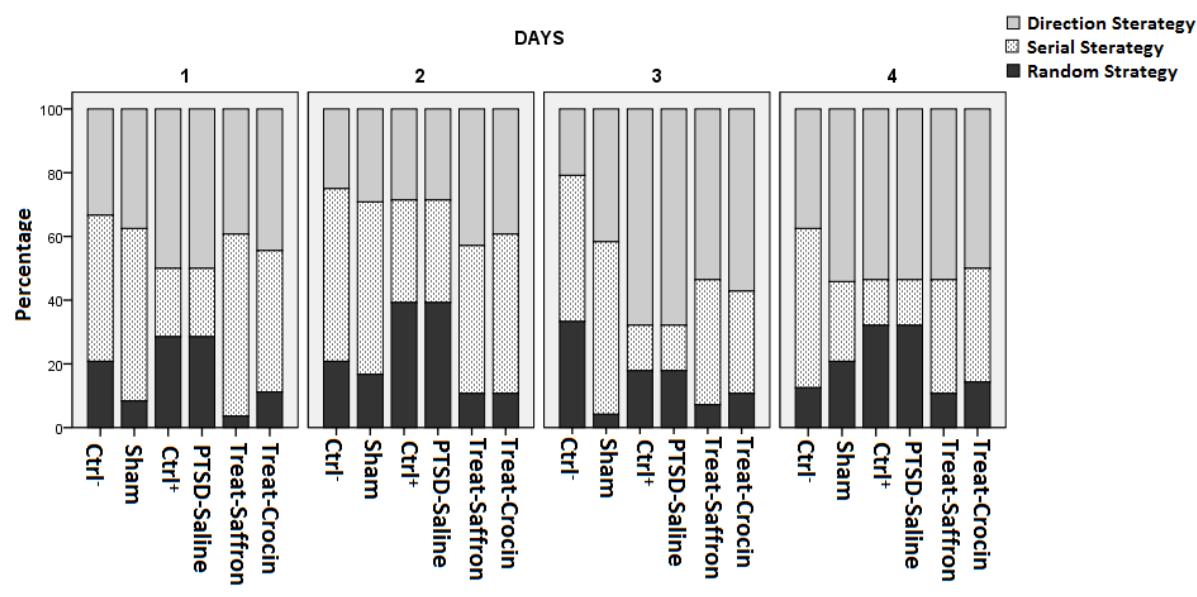

Figure 3. Changes in the search strategy and spatial learning memory were measured on day 21 post-shock in 6 groups at the re-exposure time (Direct, serial and random strategy to reach target hole). 
Effect of Saffron Aqueous Extract and Crocin on the Traveled Distance

The same results were obtained in the analysis of distance changes. However, the decreasing trend occurred in all days, independent of grouping (in all groups and all days), especially on the fourth day $(P<0.008)$. Meanwhile, there was a significant difference between the groups, independent of the days $(P<0.002)$. The difference between the two groups was measured in all days based on the Mann-Whitney test. The traveled distance in the positive group, for all days, and in the PTSD-saline group, in some days, was significantly lower than the two negative control groups $(P<0.008)$. In two treatment groups, especially saffron, the results of the traveled distance were close to the control groups. In addition, on the second, third and fourth days the two groups of intervention therapy had a significant difference with the positive groups $(P<0.002)$ (Figure 5)

\section{Effect of Saffron Aqueous Extract and Crocin on the Latency Time}

Unlike the two variables, the number of errors and traveled distance in stress groups, the latency time in stress groups was higher than the control and treatment groups. In this regard, a nonparametric test was used to evaluate the variance of time spent in the groups during the days. This is due to the fact that despite the normal distribution in the groups, Box's test reached a significant level below $P<0.0005$. The comparison of latency time in each group during the day was evaluated based on Friedman's test. There was also a decreasing trend only in the negative group and in the PTSD- saline group with $\mathrm{P}$-value $<0.004$, and $\mathrm{P}$-value $<0.02$ respectively. Yet, in other groups, there was no significant difference, except for the first day in which the saffron treatment group was significantly lower than normal saline group $(P<0.04)$.

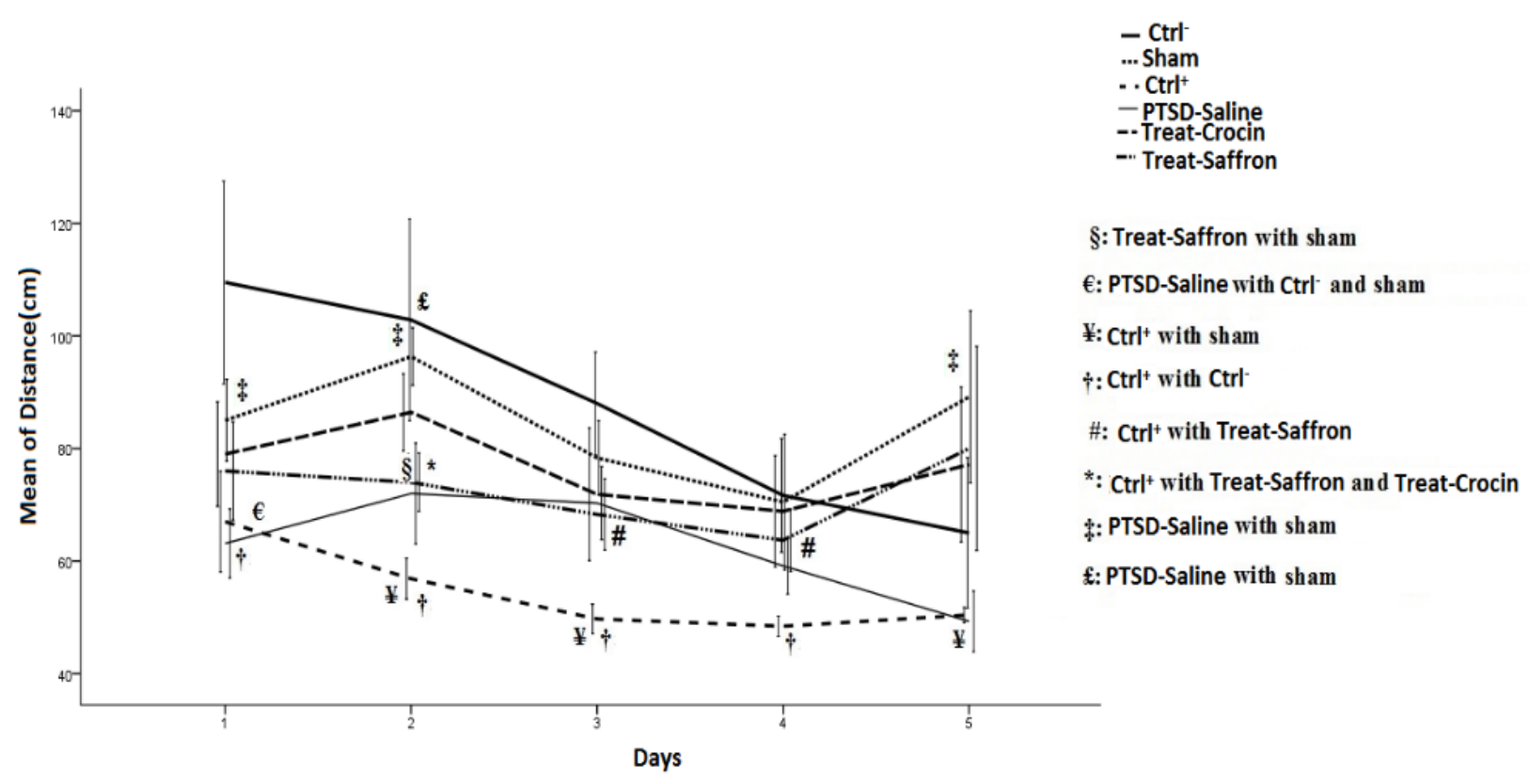

Figure 5. The effects of ICV administration of saffron aqueous extract and crocin on the traveled distance to find the target hole in the rats, 21 days after electric foot shock stress termination. There was a significant difference between the groups independent of the days $(P<0.002)$. Significant decrease occurred during the days independent of grouping, especially on the fourth day $(P<0.008)$.

\section{Discussion}

Numerous studies have been carried out on metabolic disturbance (26), memory damage, and avoidance of learning in extreme events using electric foot shock on laboratory rats (27). The current study was designed to create a post-traumatic stress disorder model in rats by an electric foot shock $(15,24)$. The results of our study indicated a reduction in corticosteroid concentrations in the animal model of PTSD. It was manifested that treatment with crocin and saffron aqueous extract significantly increased corticosterone levels at all 3 levels (basal, stress and final). Consequently, no significant difference was found between the treatment and control group. In line with our study, the decreased glucocorticoid levels and increased negative feedback were strongly associated with stress-related disorders such as PTSD $(28,29)$. This treatment probably reduces the enhancement of negative feedback of the glucocorticosteroids in the pituitary and improves the function of the HPA axis. Notably, negative feedback as a responsible factor in the glucocorticoid secretion inhibits glutamate receptors (GluR) in the pituitary, hypothalamus, and 
amygdala. However, this inhibition may sometimes not happen based on the severity of stress, and therefore, the serum glucocorticoid level remains high (29). However, this mechanism is not available in patients with PTSD; therefore, their serum and urine cortisol levels decrease $(29,30)$. Considering the inhibitory effect of saffron extract and crocin on NMDA glutamate receptors (31), it seems that there is a close mechanism between NMDA receptors and glucocorticoid in the increase of corticosteroin and the decrease of the complications of PTSD with saffron extract and crocin. However, such findings need further research performed on the types of communication in various brain regions and in more advanced models associated with PTSD. In the present study, we examined the dopaminergic-related behavioral changes (freezing) in PTSD and non-PTSD groups. The increase in freezing behavior of the PTSD model and the decrease of freezing in treatment groups receiving saffron aqueous extract and crocin were observed. One of the most important signs of fear is freezing behavior, which is due to the effect of severe amygdule inhibition in the dorsal and ventral striatum (32). It seems that in this study, saffron extract and crocin affect the amygdule moderator activity on the dorsal striatum, and thereby, reduce the freezing behavior of the animal (32). Moreover, the effects of acute stress (PTSD) and saffron extract on learning and spatial memory were assessed in the PTSD model. In this respect, significant changes in spatial learning were observed in PTSD and treatment groups compared to the negative control group. A significant difference was also observed in the traveled distance and the number of errors in 4 days between the groups. The current study showed that stress was able to reduce the number of errors and distance traveled to reach the target hole (increasing spatial memory) in the PTSD group compared to the treatment and negative control groups. This result can initially lead to a false judgment about stress performance in which stress may be considered as a possible factor involved in the cognitive function improvement of rats. A random strategy was chosen in the stress group [rather than serial and direct strategy to find the target hole, and compared to the negative control group and treatment group (saffron aqueous extract), the stress group caused a decrease in motion and anxiety-like behaviors that eventually led to a decrease in the number of errors and traveled distance in the stress groups. As a matter of fact, previous studies have illustrated that saffron and its active compounds such as crocin, can prevent memory loss and learning degradation as well as oxidative damage caused by chronic stress in the hippocampus (33). In a research reported by Pitsikasin, the antagonistic effects of a single dose injection of saffron extract on memory damage caused by a scopolamine injection were noted in the detection of objects and the step-avoidance task in the animal model (34). Notably, the NMDA receptor is known to be active in the post-training memory process in the amygdule and hippocampus (31). Hence, in the previous studies, the involvement of NMDA receptors of the effects of saffron and active components on memory was clearly reported (31). Several studies have shown that stress can stimulate memory by stimulating many pathways of dopamine, glutamate, and cholinergic in the brain (35). Our results are also in agreement with the previous results that indicate the improvement of spatial learning in the PTSD model and the model treated with saffron extract and crocin.

\section{Conclusion}

Our study showed that the saffron aqueous extract and its constituent, crocin, can alleviate the symptoms of the PTSD model of rats. Based on the obtained results, the increase in the serum corticosteron level may improve the function of the HPA axis and the freezing behavior reduction occurs probably due to the effect of saffron aqueous extract and crocin on dorsal striatum activity. Moreover, the spatial learning increase is possibly due to the effects of saffron and its active components on the improvement of memory through NMDA receptors.

\section{Acknowledgements}

This study was conducted as a part of Asalgoo's thesis project, which was supported by the Neuroscience Sciences Research Center, Baqiyatallah University of Medical Sciences, Tehran, Iran.

\section{Conflict of Interest}

Authors declare no conflict of interests.

\section{Author Contributions}

All authors have contributed to this study in terms of study design, experimental work, statistical analysis, scientific writing, and revisions.

\section{References}

1. Asalgoo S, Jahromi G, Meftahi G, Sahraei H. Posttraumatic stress disorder (ptsd): Mechanisms and possible treatments. Neurophysiol. 2015; 47(6): 482-9. [DOI:10.1007/s11062-0169559-9]

2. Association AP. American Psychiatric Association: Diagnostic and Statistical Manual of Mental Disorders: DSM-IV. Washington, DC: American Psichiatric Association. 1994.

3. Jacobson L. Hypothalamic-pituitary-adrenocortical axis regulation. Endocrinol Metab Clin. 2005; 34(2): 271-92. [DOI:10.1016/j.ecl.2005.01.003] [PMID]

4. Yehuda R. Current status of cortisol findings in post-traumatic stress disorder. Psychiat Clin North Am. 2002; 25(2): 341-68. [DOI:10.1016/S0193-953X(02)00002-3]

5. McEwen BS, Gianaros PJ. Central role of the brain in stress and adaptation: links to socioeconomic status, health, and disease. Ann N Y Acad Sci. 2010; 1186(1): 190-22. [DOI:10.1111/j.1749-6632.2009.05331.x] [PMID] [PMCID]

6. Graham BM, Milad MR. The study of fear extinction: implications for anxiety disorders. Am J P. 2011; 168(12): 125565. [DOI:10.1176/appi.ajp.2011.11040557] [PMID] [PMCID] 
7. Grillon C. Associative learning deficits increase symptoms of anxiety in humans. Biol Psychiat. 2002; 51(11): 851-8. [DOI:10.1016/S0006-3223(01)01370-1]

8. Lucassen P, Meerlo P, Naylor A, et al. Regulation of adult neurogenesis by stress, sleep disruption, exercise and inflammation: Implications for depression and antidepressant action. Europ Neuropsycho Pharmacology. 2010; 20(1): 1-17. [DOI:10.1016/j.euroneuro.2009.08.003] [PMID]

9. Lindauer RJ, Vlieger E-J, Jalink M, Olff M, Carlier IV, Majoie $\mathrm{CB}$, et al. Effects of psychotherapy on hippocampal volume in out-patients with post-traumatic stress disorder: a MRI investigation. Psychological Med. 2005; 35(10): 1421-31. [DOI:10.1017/S0033291705005246] [PMID]

10. Eichenbaum H, Cohen NJ. From conditioning to conscious recollection: Memory systems of the brain: Oxford University $\begin{array}{lll}\text { Press on } & 004 .\end{array}$ [DOI:10.1093/acprof:oso/9780195178043.001.0001]

11. Rey HG, Ison MJ, Pedreira C, et al. Single-cell recordings in the human medial temporal lobe. J Anatomy. 2015; 227(4): 394408. [DOI:10.1111/joa.12228] [PMID] [PMCID]

12. Roelofs K. Freeze for action: neurobiological mechanisms in animal and human freezing. Philos Trans R Soc Lond B Biol Sci. 2017; 372(1718): 20160206. [DOI:10.1098/rstb.2016.0206] [PMID] [PMCID]

13. Gohari AR, Saeidnia S, Mahmoodabadi MK. An overview on saffron, phytochemicals, and medicinal properties. Pharmcogn Rev. 2013; 7(13): 61-6. [DOI:10.4103/0973-7847.112850] [PMID] [PMCID]

14. Mokhtari Hashtjini M, Pirzad Jahromi G, Meftahi GH, Esmaeili $\mathrm{D}$, Javidnazar D. Aqueous extract of saffron administration along with amygdala deep brain stimulation promoted alleviation of symptoms in post-traumatic stress disorder (PTSD) in rats. Avicenna J Phytomed. 2018: 8: 358-69.

15. Asalgoo S, Tat M, Sahraei H, Jahromi GP. The psychoactive agent crocin can regulate hypothalamic-pituitary-adrenal axis

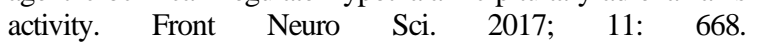
[DOI:10.3389/fnins.2017.00668] [PMID] [PMCID]

16. Hensel A, Niehues M, Lechtenberg M, Quandt B, Schepmann D, Wünsch B. Analytical and functional aspects on saffron from Crocus sativus L: development of quality control methods, species assortment and affinity to sigma-1 and NMDA receptors. Planta Medica. 2006; 72(11): 74. [DOI:10.1055/s-2006-949874]

17. Hosseinzadeh H, Ziaei T. Effects of Crocus sativus stigma extract and its constituents, crocin and safranal, on intact memory and scopolamine-induced learning deficits in rats performing the Morris water maze task. J Med Plant. 2006; 3(19): 40-50.

18. Haghighizad H, Pourmotabbed A, Sahraei H, Ghadami MR, Ghadami S, Kamalinejad M. Protective effect of Saffron extract on morphine-induced inhibition of spatial learning and memory in rat. Physiol Pharmacol. 2008; 12(3): 170-9.

19. Abe K, Saito H. Effects of saffron extract and its constituent crocin on learning behaviour and long-term potentiation.

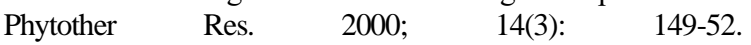
https://doi.org/10.1002/(SICI)10991573(200005)14:3<149::AID-PTR665>3.0.CO;2-5 [DOI:10.1002/(SICI)1099-1573(200005)14:33.0.CO;2-5]

20. Hadipour M, Kaka G, Bahrami F, et al. Crocin improved amyloid beta induced long-term potentiation and memory deficits in the hippocampal CA1 neurons in freely moving rats.
Synapse. 2018; 72(5): e22026. [DOI:10.1002/syn.22026] [PMID]

21. Sahraei H, Fatahi Z, Eidi A, et al. Inhibiting post traumatic stress disorder (PTSD) induced by electric shock using ethanol extract of saffron in rats. J Biol Res Thessalon. 2012; 18: 320-7.

22. Hosseinzadeh H, Jahanian Z. Effect of Crocus sativus L.(saffron) stigma and its constituents, crocin and safranal, on morphine withdrawal syndrome in mice. Phytother Res. 2010; 24(5): 72630. [DOI:10.1002/ptr.3011] [PMID]

23. Mikics E, Baranyi J, Haller J. Rats exposed to traumatic stress bury unfamiliar objects-a novel measure of hyper-vigilance in PTSD models? Physiol Bhav. 2008; 94(3): 341-8. [DOI:10.1016/j.physbeh.2008.01.023] [PMID]

24. Mokhtari hashtjini M, Pirzad Jahromi G, sadr SS, Meftahi GH, Hatef B, Javidnazar D. Deep brain stimulation in a rat model of post-traumatic stress disorder modifies forebrain neuronal activity and serum corticosterone. Iran J Basic Med Sci. 2018; 21(4): 370-5.

25. Gawel K, Gibula E, Marszalek-Grabska M, Filarowska J, Kotlinska JH. Assessment of spatial learning and memory in the Barnes maze task in rodents-methodological consideration. Naunyn-Schmiedeberg's Arch Pharmacol. 2018: 1-18. [DOI:10.1007/s00210-018-1589-y] [PMID] [PMCID]

26. Mortazaei S, Sahraei H, Bahari Z, Meftahi GH, Pirzad Jahromi G, Hatef B. Ventral tegmental area inactivation alters hormonal, metabolic, and locomotor responses to inescapable stress. Arch Physiol Biochem. 2018: 1-9. [DOI:10.1080/13813455.2018.1455711] [PMID]

27. McEwen BS. Physiology and neurobiology of stress and adaptation: central role of the brain. Physiol Rev. 2007; 87(3): 873-904. doi:10.1152/physrev.00041.2006 [DOI:10.1152/physrev.00041.2006] [PMID]

28. Daskalakis NP, Lehrner A, Yehuda R. Endocrine aspects of posttraumatic stress disorder and implications for diagnosis and treatment. Endocrinol Metab Clin. 2013; 42(3): 503-13. [DOI:10.1016/j.ecl.2013.05.004] [PMID]

29. Yehuda R. Status of glucocorticoid alterations in post-traumatic stress disorder. Ann N Y Acad Sci. 2009; 1179(1): 56-69. [DOI:10.1111/j.1749-6632.2009.04979.x] [PMID]

30. Yehuda R, LeDoux J. Response variation following trauma: a translational neuroscience approach to understanding PTSD. Neuron. 2007; 56(1): 19-32. [DOI:10.1016/j.neuron.2007.09.006] [PMID]

31. Lechtenberg M, Schepmann D, Niehues M, Hellenbrand N, Wünsch B, Hensel A. Quality and functionality of saffron: quality control, species assortment and affinity of extract and isolated saffron compounds to NMDA and $\sigma 1$ (sigma-1) receptors. Planta Medica. 2008; 74(07): 764-72. [DOI:10.1055/s-2008-1074535] [PMID]

32. Hooshmandi Z, Rohani AH, Eidi A, Fatahi Z, Golmanesh L, Sahraei H. Reduction of metabolic and behavioral signs of acute stress in male Wistar rats by saffron water extract and its constituent safranal. Pharmaceutical Biology. 2011; 49(9): $947-$ 54. [DOI:10.3109/13880209.2011.558103] [PMID]

33. Papandreou MA, Tsachaki M, Efthimiopoulos S, Cordopatis P, Lamari FN, Margarity M. Memory enhancing effects of saffron in aged mice are correlated with antioxidant protection. Behav Brain Res. 2011; 219(2): 197-204. [DOI:10.1016/j.bbr.2011.01.007] [PMID] 
34. Pitsikas N, Sakellaridis N. Crocus sativus L. extracts antagonize memory impairments in different behavioural tasks in the rat. Behav Brain Res. 2006; 173(1): 112-5. [DOI:10.1016/j.bbr.2006.06.005] [PMID]
35. Kvetnansky R, Sabban EL, Palkovits M. Catecholaminergic systems in stress: structural and molecular genetic approaches. Physiol Rev. 2009; 89(2): 535-606. [DOI:10.1152/physrev.00042.2006] [PMID]

\section{How to Cite This Article:}

Asalgoo S, Pirzad Jahromi G, Hatef B, Sahraei H. The Effect of Saffron Aqueous Extract and Crocin on PTSD

Rat Models: The Focus on Learning and Spatial Memory. J Adv Med Biomed Res. 2018; 26 (119) :34-42

\section{Download citation:}

$\underline{\text { BibTeX }}|\underline{\text { RIS }}| \underline{\text { EndNote }}|\underline{\text { Medlars }}| \underline{\text { ProCite }}|\underline{\text { Reference Manager }}| \underline{\text { RefWorks }}$

\section{Send citation to:}

(3) Mendeley 2 Zotero (:)RefWorks RefWorks 